\title{
Ribosomal RNA Synthesis in E. coli
}

DURING the past several months the idea that a factor called psi (see Nature, 228, $748 ; 1970$ ) plays a critical role in controlling the transcription of the ribosomal RNA genes of $E$. coli by RNA polymerase has met with considerable criticism, chiefly because of the discovery that the transcription of these genes in vitro appears to depend on the chemical composition of the assay medium as well as on the presence or absence of psi factor. As a result the picture of the regulation of synthesis of ribosomal RNA is today a great deal murkier than it seemed this time last year (see Nature, 228, $711 ; 1970$ ) and the function of psi factor and the nucleotide guanosine tetraphosphate (ppGpp) is very much sub judice. There can, however, be no doubt that when normal stringent $E$. coli cells are starved of essential amino-acids, RNA synthesis as well as protein synthesis is diminished, synthesis of ribosomal RNAs being especially reduced by starvation.

Irrespective of whether or not psi factor and ppGpp have anything to do with these phenomena, there are two obvious and possible ways in which the amount of ribosomal RNA, and for that matter any RNA synthesis, might be regulated. It might be controlled by altering the rate at which RNA polymerase catalyses the formation of phosphodiester bonds and hence elongates the nascent RNA chain. Alternatively the amount of RNA made might be controlled by altering the rate at which the synthesis of RNA chains is initiated, with the rate of their elongation, once the initiation step has been accomplished, remaining constant. As Stamato and Pettijohn report on page 99 of this issue of Nature New Biology, this latter seems to be the case as far as the synthesis of ribosomal RNA in stringent $E$. coli is concerned.

Using a competition hybridization technique they have established that whereas about thirty per cent of all nascent RNA molecules in mutant, relaxed $E$. coli and stringent $E$. coli growing in the presence of amino-acids is ribosomal RNA, less than seven per cent of all nascent RNA molecules in starved, stringent cells is ribosomal. And by using purified ribosomal gene DNA instead of total cell DNA in these hybridization experiments, Stamato and Pettijohn estimate that only about five per cent of the nascent RNA in starved stringent cells is ribosomal; this is presumably the basal level of transcription of the ribosomal RNA genes which continues in spite of starvation for essential amino-acids.

Within thirty to forty seconds of the restoration of amino-acids to the starved cells, incorporation of ${ }^{3} \mathrm{H}$ uridine into RNA increases rapidly, and this restoration of RNA synthesis can be blocked with rifampicin, which specifically inhibits the initiation but not the elongation of RNA chains. This observation is not, of course, consistent with the idea that in starved cells RNA polymerase molecules are arrested, having started but being unable to complete RNA molecules. Pursing this lead, Stamato and Pettijohn examined the kinetics of restoration of ribosomal RNA synthesis after adding amino-acids to starved cultures. Their results indicate that $16 \mathrm{~S}$ ribosomal RNA appears before $23 \mathrm{~S}$ ribosomal RNA as expected if, after relieving starvation, polymerase molecules have to reinitiate new rounds of ribosomal gene transcription rather than complete randomly arrested nascent chains. For not only is $16 \mathrm{~S}$ RNA smaller than $23 \mathrm{~S}$, and should therefore take less time to complete, but also, as Kossman, Stamato and Pettijohn have shown (see page 102 of this issue), the $16 \mathrm{~S}$ and 23S RNAs are probably synthesized from one transcriptional unit with the sequences for 16S RNA being proximal to the initiation point. These data all indicate that when stringent cells are starved and ribosomal RNA is depressed there is some interruption in the initiation of transcription specifically at the ribosomal RNA genes and there may, of course, also be some reduction in the rate at which these RNAs can be elongated.

Pettijohn and his colleagues and other groups also have in the past provided evidence that both the $16 \mathrm{~S}$ and $23 \mathrm{~S}$ RNAs in E. coli ribosomes are derived from precursors only slightly larger than the mature molecules to which they give rise. Whether or not these two precursor molecules in their turn are derived from a single large precursor chain containing both $16 \mathrm{~S}$ and $23 \mathrm{~S}$ sequences, as is the case in eukaryotes, is a question that has yet to be answered unambiguously. Experiments in which actinomycin D was used to analyse the pattern of inhibition of $16 \mathrm{~S}$ and 23S RNA synthesis have led some people to suggest that the two RNAs are synthesized independently. On the other hand, experiments in which rifampicin was used to inhibit the synthesis of these RNAs have led others to conclude that the two RNA species are synthesized as part of a single RNA chain. The fact that the 16S and 23S RNA genes map very close together means that this is feasible.

No doubt this issue will ultimately be resolved either by the isolation and characterization of the immediate product or products, as the case may be, of transcription or by the isolation and in vitro transcription of the ribosomal genes. That has yet to be done, but if the experiments that Kossman et al. now report, together with the results of the rifampicin experiments, are anything to go by, the betting must be that in bacteria as in eukaryotes the two ribosomal RNA genes are part of a single transcriptional unit. Kossman et al. have exploited stringent bacteria to provide a system in which initiation of ribosomal RNA synthesis can be synchronized. They have measured the times at which $16 \mathrm{~S}$ and 23S RNAs become detectable after amino-acids are restored to starved cells, and find that the former RNA always precedes the latter. This result rules out a model in which the two genes are in a single transcriptional unit with the $23 \mathrm{~S}$ gene preceding the $16 \mathrm{~S}$ gene. So either the genes must be independent or in one unit with the $16 \mathrm{~S}$ gene first. Competition hybridization experiments with nascent RNA made very soon after starved cells are re-fed reveal a predominance of $16 \mathrm{~S}$ nascent RNA chains and only at later times can comparable amounts of $23 \mathrm{~S}$ RNA be detected. This observation leads Kossman et al. to the obvious conclusion that the two ribosomal RNA genes are indeed in a single transcriptional unit in $E$. coli, that the 16S RNA gene is $5^{\prime}$ terminal, and that $16 \mathrm{~S}$ RNA may well be cleaved from the nascent product of transcription of this unit before the polymerase has had time to complete the synthesis of the $23 \mathrm{~S}$ sequence and reach the $3^{\prime}$ terminus. 